\title{
INFECTION
}

\section{Resistance to infection-skin adipocytes in the spotlight}

The number of dermal adipocytes is significantly increased in mice after skin infection with Staphyloccocus aureus, report Richard Gallo and colleagues in Science. These findings uncover a role for dermal adipose tissue in the defence against microbial infection.

"This project was inspired by the unexpected observation we made previously that the skin microbiome normally interacts with cells deep in the dermis," explains Gallo. Seeing bacteria interacting with adipocytes prompted the researchers to investigate whether adipocytes have an innate capacity to defend against infection.

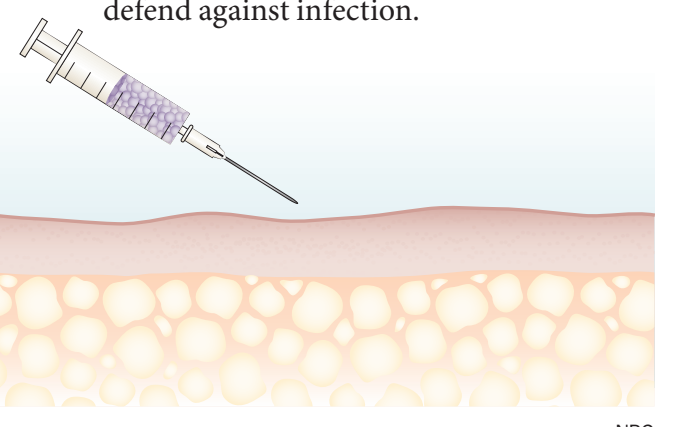

Gallo and collaborators compared the skin structure of mice injected subcutaneously with $S$. aureus, an important infectious agent in humans, and that of mice injected with a saline solution. The researchers also investigated the differentiation and proliferation of dermal adipose cells in response to infection by a combination of bromodeoxyuridinetracing experiments, analysis of a genetic mouse model of impaired adipogenesis, immunostaining and pharmacological inhibition of adipogenesis. S. aureus infection triggered rapid proliferation of preadipocytes and expansion of dermal adipose tissue. Importantly, skin infection was more severe in mice with impaired adipogenesis than in mice in which the processes of preadipocyte differentiation and adipocyte proliferation were normal.

The researchers also compared gene expression in undifferentiated preadipocytes and differentiated adipocytes in culture. The expression levels of Camp, which encodes cathelicidin antimicrobial peptide, were higher in differentiated than in undifferentiated cells. Both mRNA and protein expression of cathelicidin antimicrobial peptide were increased in the skin of infected mice, and inhibition of adipogenesis led to decreased production of this peptide.

"Our findings imply that conditions that hinder the function of dermal preadipocytes will predispose to infection," says Gallo. "As this hypothesis is consistent with clinical observations, it might have great importance for developing new strategies to improve therapy against infection in individuals with these conditions. We could even speculate that adipocyte stem cell therapy could be useful for the treatment of chronic local infections."

Joana Osório

Original article Zhang, L. J. et al. Innate immunity. Dermal adipocytes protect against invasive Staphylococcus aureus skin infection. Science 347, 67-71 (2015)

Further reading Nakatsuji, T. et al. The microbiome extends to subepidermal compartments of normal skin. Nat. Commun. 4, 1431 (2013) 